\title{
Identification of an Elasto-Viscoplastic Flow Stress in Large Strains and Large Strain Rates of a Paint Film from Impacts
}

\author{
Bassem Zouari* — Maurice Touratier** \\ * Presently at Département de Génie Mécanique \\ Ecole Nationale des Ingénieurs de Sfax \\ BP W, 3038 Sfax, Tunisia \\ bassem.zouari_ater@univ-orleans.fr \\ ** LMSP-UMR 8106 CNRS-ENSAM-ESEM \\ Ecole Nationale Supérieure d'Arts et Métiers \\ 151, boulevard de l'Hôpital \\ F-75013 Paris \\ maurice.touratier@paris.ensam.fr
}

\begin{abstract}
To achieve computer simulations of forming processes using the finite element method (FEM), we need input data such as non linear material behaviour laws and friction laws. In the majority of cases these data are very specific to the studied process because they transfer the physical phenomena acting in the studied process. Thus there are no direct experimental means that can provide us these data. Inverse problems give us an answer to this lack of input data. This consists of evaluating the parameters that would lead to the most accurate model, minimizing the difference between the experimental and its simulation results. In this paper, we propose to identify the parameters of the material behaviour law in the case of impact loading of a polyurethane paint film (PAC33) by solving the corresponding inverse problem. This inverse problem consists of adjusting the paint's behaviour law parameters in the finite element model until the calculated crater dimensions match the measured dimensions in the least square sense.
\end{abstract}

KEYWORDS: Large strain rates, Inverse problem, Finite element, behaviour law. 


\section{Introduction}

Paint stripping and the repainting of aircraft surfaces are required periodically during the lifetime of an aircraft. Traditionally, paint removal has been achieved by chemical stripping. However, the use of methylene chloride and phenol-based chemical strippers for aircraft paint removal generates large quantities of hazardous waste and creates health and safety problems for the operator. One possible alternative to chemical stripping is starch media blast cleaning, which is increasingly being used to remove organic coatings from substrates. With this paint stripping method, a stream of starch media particles removes the coating by mechanical means (Papini et al., 1997; Zouari et al., 2002).

Starch media blast cleaning is environmentally benign because the media is nontoxic and biodegradable. Due to their chemical inertness and erosion characteristics, starch media have been used for dry stripping of aircraft paint from aluminium alloys and polymer matrix composites. The process is also capable of selective stripping, removing only that needing repainting (Monnette et al., 1996). But there remains the problem of matching type of paint with the type of particle plus finding the optimal velocity and optimal impact angle to ensure maximum paint removal without damaging the substrate. The numerical simulation of this process can live up to these expectations (Zouari, 2001; Zouari et al., 2002).

Many studies have been carried out on the analysis of contact between a rigid or elastic body on a layered solid. (Komvapoulos, 1998) has used the finite element method to analyse the indentation of a layered solid in normal contact with a rigid surface. (Kral et al. 1995a, 1995b) have studied the repeated indentation of an elastic plastic layered medium by a rigid sphere. Papini and Spelt (Papini et al., 1997) developed a model for the prediction of shear stresses at the interface paint substrate. The behaviour law of the paint is assumed to be rigid plastic. The flow limit of the paint is taken equal to the dynamic hardness which is defined as the instantaneous force resisting indentation during a collision divided by the instantaneous area (Papini et al., 1998) The value of the dynamic hardness is determined by measuring the crater depth after impact. All of these studies assumed that the coating has a simplified behaviour and that it is in quasi-static state. These types of paint behaviour are not available for the simulation of the impact. We need to use a paint behaviour law which takes into account the strain rate effect on the flow limit.

Classic methods for the characterisation of the behaviour law of material (traction and flexion tests) are not useful in the case of the paint film because the latter is very thin and the strain rates acting in these tests are very small (about $10^{-2} \mathrm{~s}^{-1}$ ) compared to those acting in impact tests (about $10^{5} s^{-1}$ ).

Another method based on Hopkinson bar (Kolsky, 1963) has been used to find the flow stress characterisation required at high strain rates, for example in cutting. (Zhao, 1997) used the Hopkinson bar to identify the flow stress of polymeric foam at 
high strain rates. In general, such a test needs to design a specific specimen to be able to represent the physics considered.

To identify the behaviour law of the paint under impact, we choose here to use the inverse problem technique (Bonnet, 1999; Bui, 1993; Massoni, 1994; Tarantola, 1987) on the impact of the paint. The aim of this technique consists of comparing the experimental answers of a system and the answer of a system model to the same loading. The wanted parameters are those where the difference between experimental and model results are the smallest according to a chosen norm.

In this paper, we present the identification of paint layer behaviour law parameters by solving an inverse problem and using the finite element simulation of particle impact, based on a model including large elasto viscoplastic deformations at large strain rates. In the first section we introduce the form of the behaviour law of the paint. We then present the specific impact experiments which have been carried out in order to have experimental crater dimensions. The third section deals with the inverse problem techniques and the finite element model used in the identification procedure. Finally we present some results of the numerical simulation of paint decoating and compare these with experimental results.

\section{Constitutive law and flow stress in elasto-viscoplasticity for large plastic strains and large strain rates}

To simulate the impact of a spherical particle on a coated substrate, we first need to find a behaviour law of the coating film which takes into account the physical phenomena acting during impact and second to identify the input data of this law.

To get an idea about the behaviour of the paint film, we have done classical traction tests on it. The results of these tests were not satisfactory because we found a fragile fracture of the film and visco-elastic behaviour (Zouari, 2001). In fact in these tests the strain rates were about $10^{-2} s^{-1}$, completely different from the values reached under impact (about $10^{5} s^{-1}$ ). In this section we present the form of the constitutive law of the paint and its mathematical formulation.

The current configuration is chosen in Cartesian coordinates $x_{i} ; i=1,2,3$. To study the material behaviour under large strains and large strain rates, we introduce an elasto-viscoplastic constitutive model and the assumption that the elastic strains are small. Therefore the Eulerian strain rate tensor $\dot{\varepsilon}$ can be decomposed into its elastic part $\dot{\varepsilon}^{e l}$ and its viscoplastic part $\dot{\varepsilon}^{v p}$ so that:

$$
\dot{\varepsilon}=\dot{\varepsilon}^{e l}+\dot{\varepsilon}^{v p}
$$

where the strain rate tensor $\dot{\varepsilon}$ is defined by the symmetric part of the velocity gradient as: 


$$
\dot{\varepsilon}=\frac{1}{2}\left(\nabla \mathbf{v}+\nabla^{T} \mathbf{v}\right)
$$

In equation [2], $\nabla$ is the gradient operator in current configuration, while $\mathbf{v}$ is the Eulerian velocity vector.

In general the elastic deformation rate components $\dot{\varepsilon}^{e l}$ can be related to the Cauchy-stress rate components $\sigma_{i j}$ according to the generalised Hook's law available for a linear and isotropic thermo-elastic material as follows:

$$
\dot{\varepsilon}_{i j}^{e l}=\frac{1+v}{E} \dot{\sigma}_{i j}-\frac{v}{E} \operatorname{Tr}(\dot{\sigma}) \delta_{i j}+\alpha\left(\dot{T}-\dot{T}_{0}\right) \delta_{i j}
$$

where: $v$ is the Poisson ratio, $E$ the Young modulus, $\alpha$ the thermal dilatation coefficient, $T$ the temperature, $T_{0}$ the initial temperature, $\delta_{i j}$ the Kronecker symbol and $\operatorname{Tr}$ the trace operator. The time derivative of the stress tensor is here based on the objective Jaumann formulation using the antisymmetric part of the velocity gradient. The visco-plastic strain rate components are related to the Cauchy stress components from the classic flow rate given by:

$$
\dot{\varepsilon}_{i j}^{v p}=\lambda_{p} \frac{\partial f}{\partial \sigma_{i j}}
$$

According to the Von-Mises criterion available for isotropic materials, the viscoplastic yield function $f$ is described by:

$$
\begin{aligned}
f(\bar{\sigma}, \bar{\varepsilon}, \dot{\bar{\varepsilon}}, T, \ldots) & =\left\{\frac{3}{2} \operatorname{Tr}\left(\sigma-\frac{1}{3} \operatorname{Tr}(\sigma) I\right)^{2}\right\}^{\frac{1}{2}}-\bar{\sigma}(\bar{\varepsilon}, \dot{\bar{\varepsilon}}, T, \ldots) \\
& =\sigma_{e q}-\bar{\sigma}
\end{aligned}
$$

In equation [5], $\sigma_{e q}$ is the Von Mises equivalent stress, $\bar{\sigma}$ is the flow stress, $\bar{\varepsilon}=\int_{0}^{t} \dot{\bar{\varepsilon}} d \tau$ is the cumulated equivalent plastic strain, $\dot{\bar{\varepsilon}}$ is the generalised strain rate deduced from the second invariant of the visco-plastic strain rate tensor and $I$ is the identity operator. Taking into account the consistency condition in standard associated plasticity $\lambda_{p}=\dot{\bar{\varepsilon}} /(4 \bar{\sigma})$, we find that:

$$
\dot{\varepsilon}_{i j}^{v p}=\frac{3}{2} \frac{\dot{\bar{\varepsilon}}}{\bar{\sigma}}\left(\sigma_{i j}-\frac{1}{3} \operatorname{Tr}(\sigma) \delta_{i j}\right)
$$


Finally, equations [1], [3] and [6] give us the standard elasto-visco-plastic constitutive law expressed in the current configuration.

Our objective is now to express the flow stress $\bar{\sigma}$ by introducing physical features required by simulation of paint decoating by impacts. In this case strain rates in the range of $10^{3}-10^{5} \mathrm{~s}^{-1}$ occur. The constitutive law of the paint must take into account the effect of strain rates. In order to include sensitivity to the above phenomena, the flow stress of the paint is modelled by the following modified Johnson-Cook law (Johnson et al., 1983):

$$
\bar{\sigma}=A\left(I+C \ln \dot{\bar{\varepsilon}}^{*}\right)
$$

where $\dot{\bar{\varepsilon}}^{*}=\dot{\bar{\varepsilon}} / \dot{\varepsilon}_{0}$ is the dimensionless plastic strain rate for $\dot{\varepsilon}_{0}=1 \mathrm{~s}^{-1}, A$ the elastic limit of the material and $C$ the viscosity.

The unknown parameters $A$ and $C$, are subjected to constraints based on the physics of the problem. These constraints must deal with the thermal activation theory developed by Eyring (Brown, 1986). For polyurethane, they must satisfy the conditions (Van Krevelen, 1990):

$$
\left\{\begin{array}{l}
A-0.01 G P a \geq 0 \\
2 G P a-A \geq 0 \\
C-0.012 \geq 0 \\
0.21-C \geq 0
\end{array}\right.
$$

\section{Test bench for impacts and experiments on painted substrate}

To obtain data for the constitutive law coefficients of the paint, within impact loading conditions, we have to solve an inverse problem (Bonnet, 1999; Bui 1993; Massoni, 1994; Tarantola, 1987). The aim of this technique is to compare the impact crater dimensions issued from experiments with those from finite element computations achieved as stated hereafter in Section 4.

\subsection{The experimental test bench}

An experimental test bench has been built (Zouari, 2001) based on a gas gun used to accelerate spherical particles. The velocity of the particles is measured with an accuracy of $3 \%$ by timing their flight between two infrared light beams located near the muzzle of the gun. The specimen is placed on a pivotal support allowing a change of the value of the impact angle. 


\subsection{Impact tests and crater measurements}

The tests consist of impacting specimens at right angles and measuring the resulting crater dimensions. Specimens used were aluminium alloy circular plates $30 \mathrm{~mm}$ in diameter and $1 \mathrm{~mm}$ thick, coated with a $0.1 \mathrm{~mm}$ polyurethane paint layer, and clamped at their circumference.

We made two right angle impacts. In the first, the particle was of alumina, had a $2 \mathrm{~mm}$ diameter and an initial velocity of $45 \mathrm{~m} / \mathrm{s}$. In the second, the particle was of steel (100C6), had a $2 \mathrm{~mm}$ diameter and an initial velocity of $35 \mathrm{~m} / \mathrm{s}$. In the two cases of impact, the paint was not allowed to delaminate nor crack because in our finite element model we assumed that the paint is subjected to only plastic deformation.

A crater in a paint layer is characterized by its depth (p), diameter (d) and lip height (h), see Figure 1. Before the measurement of the crater dimensions of a specimen, we examined the specimen under the microscope to see if cracks or paint delamination have occurred under impact. If there was no crack or paint delamination, then we measured the crater dimensions and the test was validated. Figure 2 represents an example of an observed crater. The measurement of crater dimensions was made with a profilometer which gave the profile of the crater with a precision equal to some micrometers.

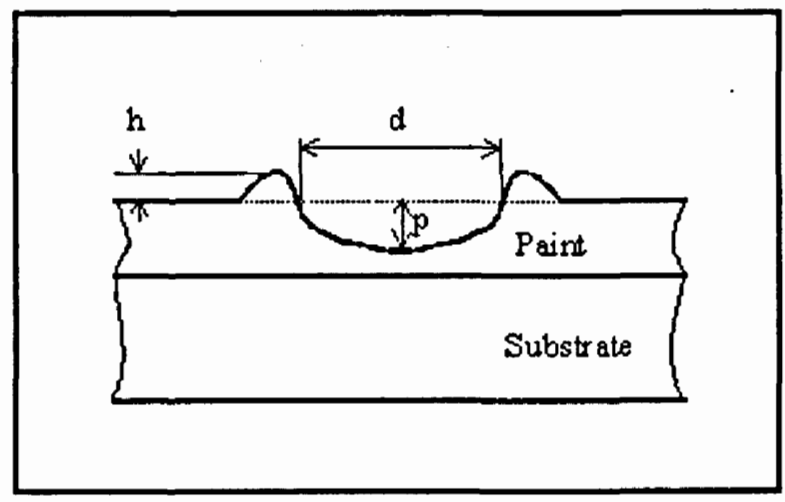

Figure 1. Crater dimensions

Table 1 shows the mean values of the crater dimensions for the two tests.

Table 1. Crater dimensions

\begin{tabular}{|c|c|c|}
\hline Particle & Steel $(100 \mathrm{C} 6)$ & Alumina \\
\hline Velocity $(\mathrm{m} / \mathrm{s})$ & 35 & 45 \\
\hline $\mathrm{h}(\mu \mathrm{m})$ & 14 & 12 \\
\hline $\mathrm{p}(\mu \mathrm{m})$ & 43 & 41 \\
\hline $\mathrm{d}(\mu \mathrm{m})$ & 580 & 560 \\
\hline
\end{tabular}




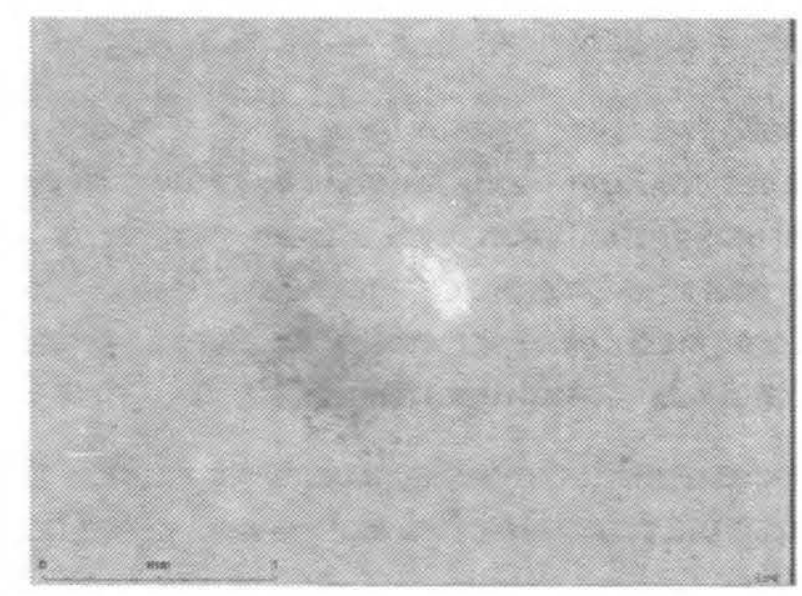

Figure 2. Microscopic observation of crater

\section{Identification of the parameters of the flow stress from impacts on paint}

In this section we solve an inverse problem which consists in minimising, in the least square means, the difference between crater dimensions issuing from experiments and those issuing from a model. The model function is what ties the crater dimensions to the coefficients of the paint constitutive law. It is evident that such a function is defined in an implicit manner as the mechanical problem is nonlinear and does not have an analytical solution. To reach this goal, we used the finite element method so as to have an estimation of the crater dimensions.

\subsection{The finite element model}

The mechanical problem consists of the simulation of a spherical particle impact on an aluminium alloy plate (A2024) coated with a polyurethane paint (PAC33). The thickness of the plate and paint layer are respectively $1 \mathrm{~mm}$ and $0.1 \mathrm{~mm}$. The plate is circular, its radius is $15 \mathrm{~mm}$, and is assumed to be clamped on at its circumference. The particle is assumed to be elastic and to have an initial velocity just before being in contact with paint. This problem is quite complex, with large deformations, high strains, non linear constitutive law and contacts. In our numerical simulation, we use the dynamic explicit finite element software ABAQUS/Explicit.

The paint constitutive law is given by equation [7], which is the Johnson Cook law (Johnson et al., 1983) where we neglect the effect of the plastic strain on the flow limit. For the substrate we used an elastic perfectly plastic law with a yield stress equal to $465 \mathrm{MPa}$. The Young's modulus of the substrate is $71 \mathrm{GPa}$. The Young's modulus of the paint is $2.3 \mathrm{GPa}$. It has been obtained from traction tests on paint films at several strain rates $\left(10^{-4}-10^{-2} \mathrm{~s}^{-1}\right)$. Prony's series have been introduced to deduce the relaxation function (Zouari et al., 2000). 


\subsubsection{The mechanical problem}

In a nonlinear analysis the equilibrium of the body considered must be established in the current configuration, which is unknown. In general, it is necessary to employ an incremental formulation, and a time variable is used to conveniently describe the loading and the motion of the body (Bathe, 1996). The aim is to evaluate the equilibrium positions of the complete body at discrete time steps $0, \Delta t$, $2 \Delta t, 3 \Delta t, \ldots$, , where $\Delta t$ is the time increment.

In a Lagrangian incremental analysis approach we express the equilibrium of the body at time $t+\Delta t$ using the principle of virtual powers given below:

$$
\begin{aligned}
& \int_{l+\Delta V}^{t+\Delta t} \sigma_{i j} \dot{d}_{i j} d^{t+\Delta t} V={ }^{t+\Delta t} R \\
& { }^{t+\Delta t} R=\int_{t+\Delta V}\left({ }^{t+\Delta t} f_{i}^{B}-^{t+\Delta t} \rho^{t+\Delta t} \ddot{x}_{i}\right) \ddot{u}_{i}^{*} d^{t+\Delta t} V+\int_{t+\Delta S}{ }^{t+\Delta t} f_{i}^{S} \ddot{u}_{i} d^{t+\Delta t} S
\end{aligned}
$$

where

${ }^{i+\Delta t} \sigma_{i j}$ are the Cartesian components of the Cauchy stress tensor

$u_{i}$ are the components of the virtual velocity vector imposed on a configuration at time $t+\Delta t$

${ }^{t+\Delta t} x_{i}$ and ${ }^{t+\Delta t} \ddot{x}_{i}$ are the Cartesian co-ordinates and acceleration of a material point at time $t+\Delta t$

$$
\dot{d}_{i j}=\frac{1}{2}\left(\frac{\partial \dot{u}_{i}}{\partial^{1+\Delta} x_{j}}+\frac{\partial^{*} \dot{u}_{j}}{\partial^{i+\Delta} x_{i}}\right) \text { are the virtual strain rate tensor corresponding to the }
$$

virtual velocity field.

${ }^{t+\Delta t} V$ is the volume at time $t+\Delta t$

${ }^{r+\Delta s} f_{i}^{B}$ are the components of externally applied forces per unit volume at time $t+\Delta t$

${ }^{t+\Delta t} \rho$ is the mass density at time $t+\Delta t$

${ }^{i+\Delta} f_{i}^{S}$ are the components of externally applied surface traction per unit surface area at time $t+\Delta t$

${ }^{t+\Delta} S_{f}$ is the surface at time $t+\Delta t$ on which external tractions are applied

$\ddot{u}_{i}=\dot{u}_{i}$ evaluated on the surface ${ }^{i+\Delta t} S_{f}$ 
An approximate solution of equation [9] can be obtained by referring all variables to a previously calculated known equilibrium configuration and linearising the resulting equation, see reference (Bathe, 1996).

\subsubsection{The finite element discretisation}

To solve equation [9], we used the finite element method for the space discretisation. Equation [9] written at time $t$ and discretized by finite elements then becomes (Marusich et al., 1995):

$$
M^{\prime} \ddot{x}+{ }^{\prime} F_{\text {int }}={ }^{\prime} F_{e x t}+{ }^{\prime} F_{c}
$$

where,

$\boldsymbol{M},{ }^{\prime} \boldsymbol{F}_{e x t},{ }^{\prime} \boldsymbol{F}_{\text {int }}$ and ${ }^{\prime} \boldsymbol{F}_{c}$ are the diagonal mass matrix, the external force array, the internal force array and the contact force array respectively.

\subsubsection{Time integration}

To predict the displacement vector at time $t+\Delta t$, we used the second order accurate central difference scheme (Hughes et al., 1983).

$$
\begin{aligned}
& { }^{\prime} \ddot{x}=M^{-1}\left({ }^{\prime} F_{e x t}-F_{\text {int }}\right) \\
& { }^{t+\frac{1}{2}} \dot{x}={ }^{t-\frac{1}{2}} \dot{x}+{ }^{\prime} \ddot{x} \Delta t \\
& { }^{t+\Delta} x={ }^{t} x+{ }^{t+\frac{1}{2}} \dot{x} \Delta t
\end{aligned}
$$

To assure the stability of the central difference scheme, the time step must be smaller than a critical value, see reference (Hallquist, 1998).

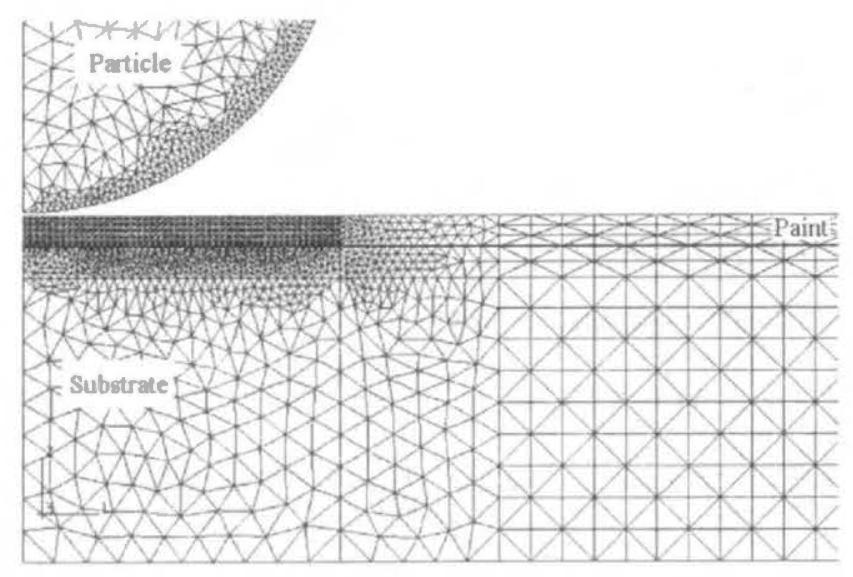

Figure 3. The mesh used in the finite element simulation 
The problem to be solved is axisymmetric, and triangular elements have been used with a reduced integration scheme. Figure 3 shows the mesh.

\subsection{Non linear least square method}

The parameters $A$ and $C$ in the finite element model are adjusted from the Levenberg-Marquardt until the calculated crater dimensions match the measured dimensions, in the least square sense. This is accomplished by minimizing the following error function, $\Phi$, with respect to the parameters $m$ :

$$
\Phi(m)=\sum_{i=1}^{2}\left[\left(1-\frac{h_{i}}{\hat{h}_{i}}\right)^{2}+\left(1-\frac{p_{i}}{\hat{p}_{i}}\right)^{2}+\left(1-\frac{d_{i}}{\hat{d}_{i}}\right)^{2}\right]
$$

where $\hat{h}_{i}, \hat{p}_{i}$ and $\hat{d}_{i}$ are experimental crater dimensions issued from the ith measure, and $h_{i}, p_{i}$ and $d_{i}$ are crater dimensions deduced from finite element simulations of the corresponding impact and $\boldsymbol{m}^{T}=\{A, C\}$ is the model parameters vector.

Because the finite element model is non linear with respect to the parameters, the minimization is referred to as a non linear least square problem.

The minimization of equation [14] is subjected to constraints on the unknowns $A, C$ based on the physics of the problem. These constraints are written in the form:

$$
\chi_{j}(m) \geq 0
$$

The constraints are enforced by the interior penalty function method, which is an unconstrained sequential minimization technique, and they are incorporated straight into the penalized multicriteria objective function $\Phi^{*}$, as follows:

$$
\Phi^{*}(m)=\Phi(m)+\sum_{j=1}^{q} \zeta_{j}(m)
$$

where the weighted penalty functions $\zeta_{j}$, are the inverse barrier functions proposed by (Carroll, 1961), and are expressed by:

$$
\zeta_{j}(m)=\frac{\omega_{j}}{\chi_{j}(m)}
$$


in which the weighting coefficients $\omega_{j}$ have to be adjusted. The advantage of the penalty function method is that the constrained problem is solved as unconstrained through the minimization of equation [16]. To minimize the objective function we used the modified Levenberg-Marquardt method (Marquardt, 1963; Schnur et al., 1992).

The aim of this method is making a first order Taylor development of the observation vector $\boldsymbol{q}^{T}=\left\{h_{1} h_{2} p_{1} p_{2} d_{1} d_{2}\right\}$ near $\tilde{\boldsymbol{m}}$ :

$$
q=q(\tilde{m})+\frac{\partial q}{\partial m} d m
$$

We note by $J=\frac{\partial q}{\partial m}$ the Jacobian matrix of the error function $\Phi$, $g=-\sum_{j=1}^{q} \frac{\partial \zeta_{j}}{\partial d m}$ and $H=\sum_{j=1}^{q} \frac{\partial^{2} \zeta_{j}}{\partial^{2} d m}$ are, respectively the first and second derivatives

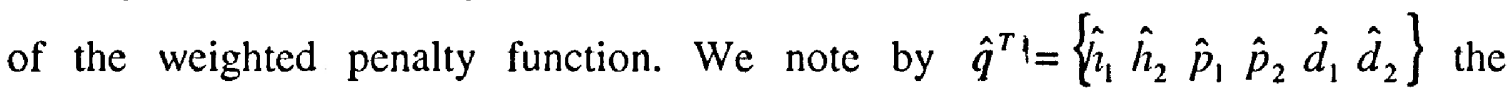
experimental observation vector and $\boldsymbol{r}=\boldsymbol{q}-\hat{q}$ the residue vector.

We write the vector $b=-J^{T} r+g$ and the matrix $L=J^{T} J+H$.

The new expression of the objective function is then given by the following expression:

$$
\begin{aligned}
& \Phi *(\tilde{m}+d m)=\frac{1}{2}[q(\tilde{m})-\hat{q}+J d m]^{T}[q(\tilde{m})-\hat{q}+J d m]+ \\
& \sum_{j=1}^{q} \zeta_{j}(\tilde{m})-d m^{T} g+\frac{1}{2} d m^{T} H d m
\end{aligned}
$$

The observation function is non linear, therefore the minimisation of the objective function is done under the constraint $\|d m\|=\left\|d m_{0}\right\|$, to ensure that the Taylor development is still available for the new parameters.

Using the Lagrange multiplier, (Marquardt, 1963) demonstrates that the solution of the problem minimisation is given by solving the following linear problem:

$$
(L+\mu I) d m=b
$$

where $\mu$ is the Levenberg-Marquardt parameter which determines the norm and direction of the step $d m$. 
To facilitate the choice of $\mu$ and to improve the conditioning of the system, equation [20] is scaled before it is solved. The scaled matrix $\underline{L}$ and the scaled vector $\underline{b}$ are defined as (Schnur et al., 1992):

$$
\begin{cases}\underline{L}_{\alpha \beta}=\frac{L_{\alpha \beta}}{\sqrt{L_{\alpha \alpha} L_{\beta \beta}}} & \alpha, \beta=1, n \\ \underline{b}_{\alpha}=\frac{b_{\alpha}}{\sqrt{L_{\alpha \alpha}}} & \alpha=1, n\end{cases}
$$

The scaled form of equation [20] is solved for $\underline{d m}$ :

$$
(L+\mu I) \underline{d m}=\underline{b}
$$

and $d m$ is calculated from $\underline{d m}$ using:

$$
d m_{\alpha}=\frac{d m_{\alpha}}{\sqrt{L_{\alpha \alpha}}} \quad \alpha=1, n
$$

Note that $\underline{L}_{\alpha \alpha}=1, \alpha=1, n$, allowing $\mu$ to be controlled in the manner of an independent problem, consistent from one iteration to the next.

Because the parameters are included implicitly in the finite element formulation, a finite difference approximation of the Jacobian matrix $J$ in equation [19] is chosen. The Jacobian matrix is calculated at each iteration by perturbing each parameter and only one at once and solving a direct finite element problem for each perturbation of the parameters.

The modified Levenberg-Marquardt method and the finite element analysis are integrated in a solution algorithm as follows:

1. Set the initial values of the parameters $m^{(0)}$ and the Levenberg-Marquardt parameter, $\mu^{(0)}=0.01$.

2. Solve the direct finite element problem and calculate the observed values $q^{(0)}$ and evaluate the error function $\Phi^{(0)}$.

3. Set the initial penalty function weight $\omega_{j}^{(0)}=0.0001 \chi_{j}^{(0)} \Phi^{(0)} ; j=1, q$. Evaluate the weighted penalty function, $\zeta_{j}^{(0)} ; j=1, q$ and the objective function $\Phi^{*(0)}$.

4. For iteration $k=0,1, \ldots, K$ approximation.

a. Calculate the Jacobian function, $J^{(k)}$ using a finite difference 
b. Calculate the penalty function derivatives to construct $H^{(k)}$ and $g^{(k)}$.

c. Solve equation $\left(\underline{L}^{(k)}+\mu^{(k)} \boldsymbol{I}\right) d \underline{\boldsymbol{m}}^{(k)}=\underline{\boldsymbol{b}}^{(k)}$, and update the parameters : $m^{(k+1)}=m^{(k)}+d m^{(k)}$.

d. Solve the direct finite element problem and calculate the new observed values corresponding to the parameters $m^{(k+1)}$. Evaluate $\zeta_{j}^{(k+1)} ; j=1, q$ and $\Phi^{*(k+1)}$.

e. Check if $\Phi^{*(k+1)}<\Phi^{*(k)}$

e.l. If false, increase $\mu^{(k)}$ and go to $4 . c$

$$
\mu^{(k)}=v \mu^{(k)} \text { with } v>1
$$

e.2. If true, continue

f. Decrease $\mu^{(k)}$ and $\omega_{j}^{(k)}$

$$
\mu^{(k+1)}=\mu^{(k)} / v, \omega_{j}^{(k)}=\omega_{j}^{(k)} / v
$$

g. Check if iteration has converged $\frac{\left|d m_{i}^{(k)}\right|}{\gamma+\left|m_{i}^{(k)}\right|}<\varepsilon, \quad i=1, n$ or $k=$ itermax

g.l. If false, then $k=k+1$ and go to $4 . a$

g.2. If true, STOP.

In this algorithm, we impose two stop criteria. The first is when the variation of each parameter becomes negligible ( $\varepsilon$ and $\gamma$ are two small positive real numbers equal respectively to $10^{-4}$ et $10^{-5}$ ). The second criterion is the number of iterations, which do not exceed a maximum value denoted by itermax.

The initial value of the Levenberg-Marquardt parameter is taken as 0.01 and $v=10$.

\subsection{Results}

We have studied the influence on the optimization results, of the initial parameters and errors of the measured dimensions of the craters.

\subsubsection{Influence of the initial parameters}

The final result of an optimization problem is very sensitive to the values of the initial parameters. To be sure that the obtained result is an absolute minimum of the objective function, we need to vary the values of the initial parameters in the optimization procedure. 
Curves in Figures 4 and 5 represent respectively the evolution of the model parameters and the value of the objective function at the end of each iteration for different values of the initial parameters.

For all initial parameter values, the objective function reaches approximately the same final value (Figure 5), but for the model parameters we found different values when the initial parameters had been changed (Figure 4). This shows that the objective function presents different local minima.

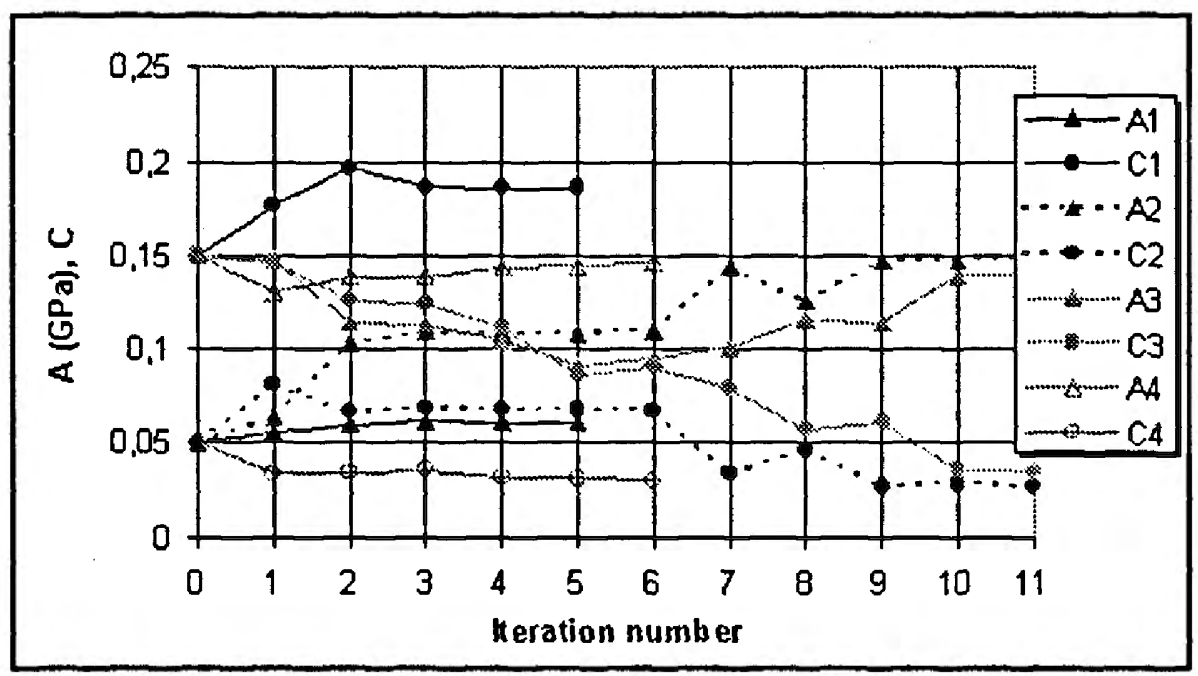

Figure 4. Evolution of $A$ and $C$ parameters with iteration numbers for different initial values

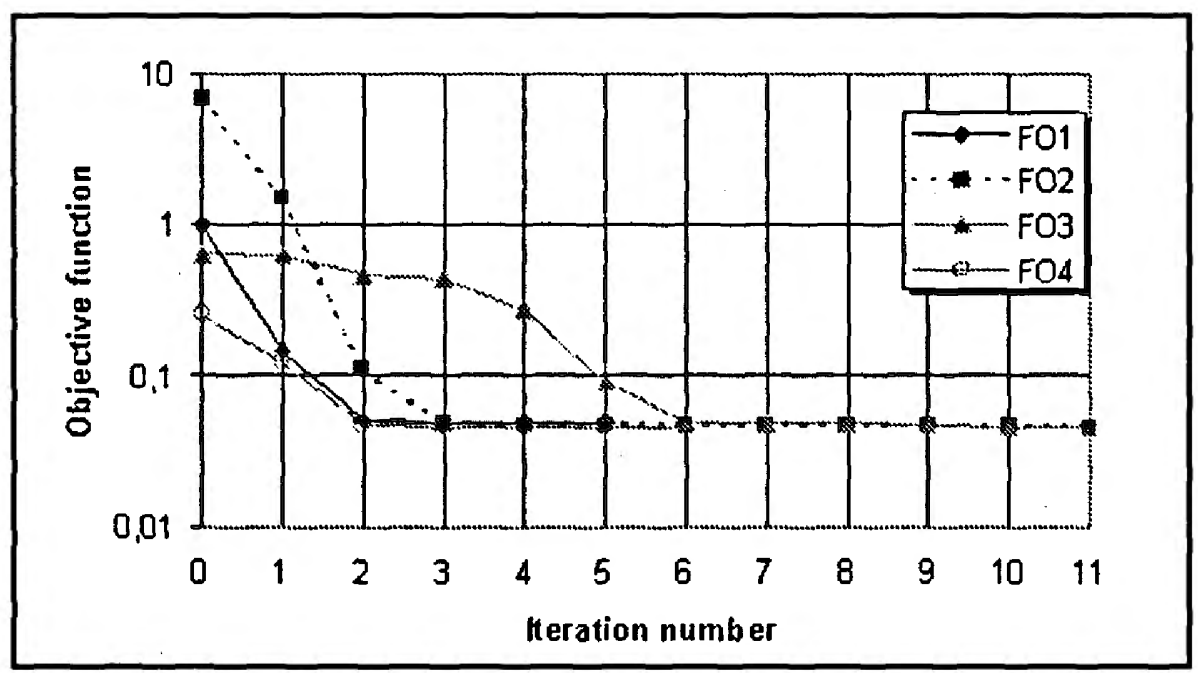

Figure 5. Evolution of the objective function with iteration numbers for different initial values 
For the three last values of the initial parameters, we obtain final values of the model parameters which are very close. The retained values for the two parameters are $A=1.4910^{-1} \mathrm{GPa}$ and $C=2.7110^{-2}$. In this optimization process, we have taken into account only three parameters of the crater and not the total. In order to see the influence of the optimization on the total crater shape, we have in Figure 6 drawn the optimized profile, the experimental profile and the profile issuing from the following initial parameters ( $A=0.510^{-1} \mathrm{GPa}$ and $C=0.510^{-1}$ ). For this test, a steel particle impact with an initial velocity of $35 \mathrm{~m} / \mathrm{s}$ has been considered. Note that similar results regarding the predicted crater shape, have been obtained in the case of a velocity of $45 \mathrm{~m} / \mathrm{s}$ and an alumina particle. We conclude that the final profile is near the experimental only on the crater flank.

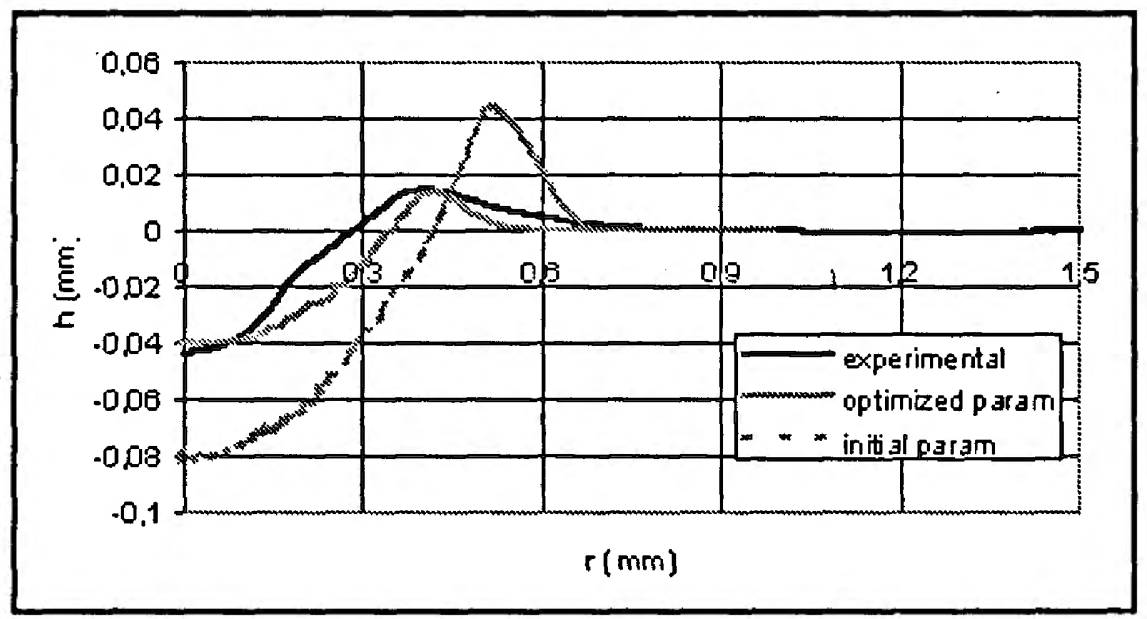

Figure 6. Crater shape before and after optimization

\subsubsection{Influence of measurement errors}

Because inverse problems are ill-posed, solutions obtained using exact data are not necessarily representative of those deduced from data including errors. In practice, errors in experimental data occur, and the stability of the solution method with respect to small changes in the given data is important. To simulate experimental errors, small and normally distributed random numbers were added to the crater dimensions. The mean of each distribution was zero, and the standard deviation was one per cent (referred to as the per cent error) of the mean of the crater dimensions. The optimized parameters obtained from each experimental data are plotted in Figure 7.

To get better information about the variation of model parameters, we calculated the relative error and the standard deviation of each model's parameters. These results are shown in table 2 . We conclude that we have better precision on parameter $A$ than on parameter $C$. 


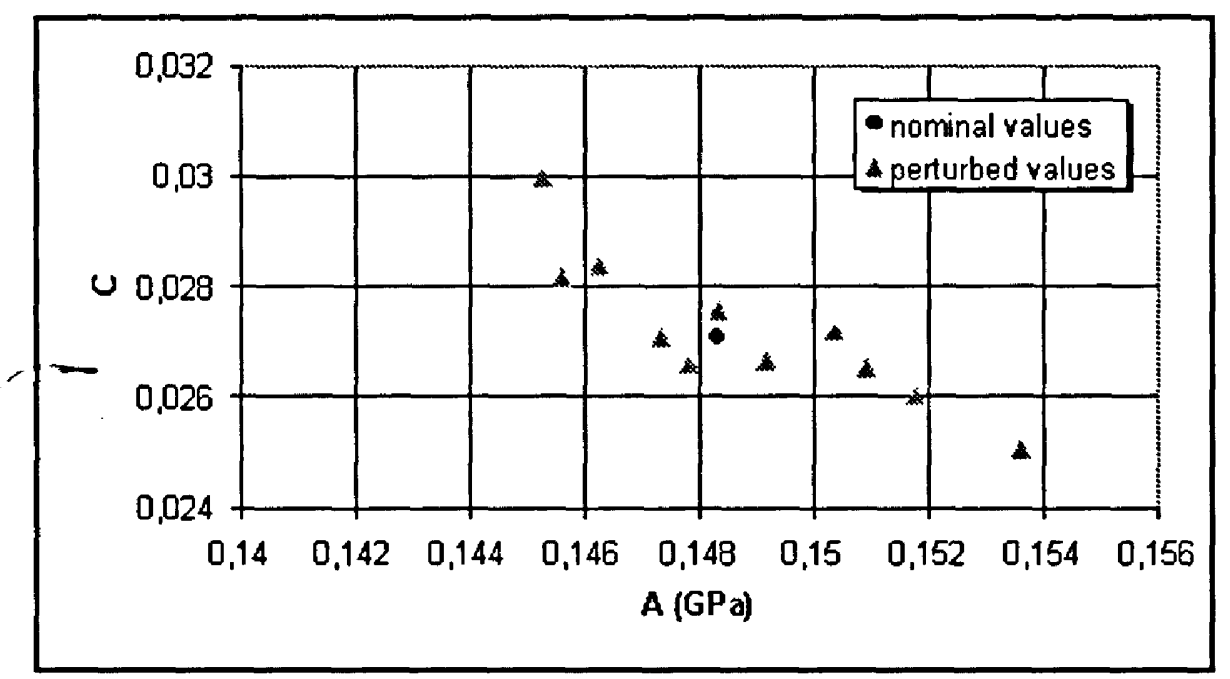

Figure 7. Model parameters for the perturbed experimental values

Table 2. Values and errors on the model parameters

\begin{tabular}{|c|c|c|c|c|}
\hline Parameter & Mean value & Absolute error & Relative error & $\begin{array}{c}\text { Standard } \\
\text { deviation }\end{array}$ \\
\hline $\mathrm{A}(\mathrm{GPa})$ & 0.14876 & $8.3210^{-3}$ & $5.6 \%$ & $2.6710^{-3}$ \\
\hline $\mathrm{C}$ & 0.02719 & $4.9410^{-3}$ & $18.2 \%$ & $1.3210^{-3}$ \\
\hline
\end{tabular}

\section{Numerical simulation of organic coating removal by particle impact}

Delamination and spalling of coatings subjected to compressive stresses have been studied by many authors. (Evans et al., EVA 1984) have developed a model based on the fracture mechanics theory and the plate buckling theory. The authors demonstrate that the delamination of pre-compressed films occurs if the film buckles, while a stress concentration appears at the buckle perimeter. (Yin, 1985) has used an iterative procedure based on the fourth-order Runge-Kutta integration formula to generate a family of nondimensionalized postbuckling solutions of the von Karman's nonlinear plate theory. These solutions determine the change of the energy-release rate with the growth of a circular thin-film or midplane delamination, which in turn determines the stability characteristics of growth.

When a coating is subjected to an indentation, the corresponding penetration into the coating induces compressive stresses. These stresses, in the presence of the initial delaminated region of the coating, can induce buckling of the coating and subsequently the propagation of delamination (Papini et al., 1998). Papini and Spelt (Papini et al., 1998) developed a model for predicting the coating buckling under normal impact by a spherical particle. They assumed that the region of the coating in contact with the particle can not buckle. 
In this section we model and simulate the delamination of the paint layer induced by the impact of a spherical particle on an aluminium alloy plate (A2024) coated with a polyurethane paint (PAC 33) in the cases of normal and oblique impact. The paint has the mechanical properties identified in the section below.

\subsection{The finite element model}

The impact problem has a symmetric plane, so we only consider half of both the particle and plate. The region under the impact (around three times the diameter of the particles) is meshed with three-dimensional eight node elements (HEXA8), see Figure 8 , as the impact is very localised, while the rest of the plate is meshed with four node Belytchko shell elements (Hallquist, 1998). The junction between the two types of mesh is achieved by introducing kinematic conditions. During the calculation, brick nodes located at the junction surface are constrained to lie along the full fiber thickness of the shell, having the capability to move relative to each other in this fiber direction.

The target elements have a full integration scheme to avoid possible hourglass modes during simulations. The other elements have a reduced integration scheme. In our model, there are 46000 brick elements and 1600 shell elements, giving 206514 degrees of freedom. In our numerical simulation, we used the dynamic explicit finite element software LS-DYNA.

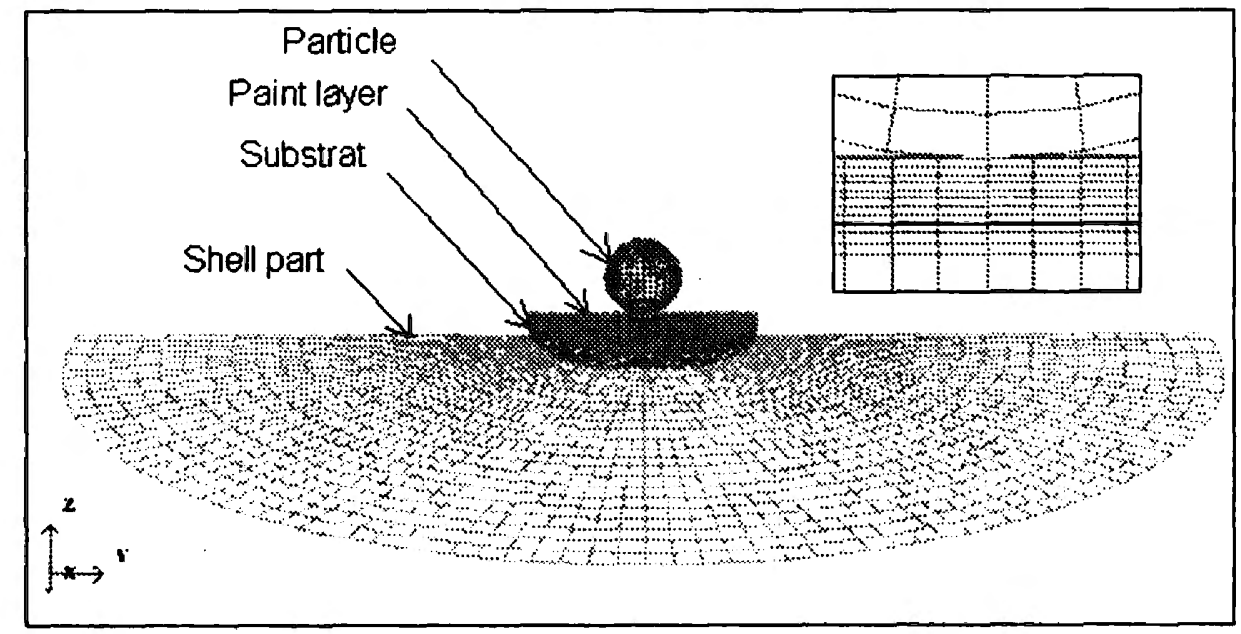

Figure 8. The 3D mesh used

In order to study the propagation of paint delamination under particle impacts, we have introduced a debonding criterion between paint and substrate in our finite element model. We adopt a special finite element mesh. Initially, corresponding nodes on the substrate surface and on the paint surface are perfectly bonded and therefore have identical displacements. During the simulation, the nodes debond 
introducing tear-off of the paint layer. This node separation is governed by the following debonding criterion,

$$
\left(\frac{\left\langle\sigma_{n}\right\rangle}{N F L S}\right)^{2}+\left(\frac{\left|\sigma_{s}\right|}{S F L S}\right)^{2} \leq 1
$$

where $\left\langle\sigma_{n}\right\rangle$ is the positive part of the stress vector normal to the interface, $\sigma_{s}$ is the shear stress, NFLS and SFLS are, respectively, normal and shear failure stresses. Failure is assumed if the left side of [24] is greater than one.

The values of $N F L S$ and SFLS are deduced from tear-off tests (Zouari, 2001) and are, respectively, equal to $50 \mathrm{MPa}$ and $200 \mathrm{MPa}$. This criterion does not take into account the results of the fracture mechanics theory.

\subsection{Results}

We have applied this model for a $81 \mathrm{~m} / \mathrm{s}$ velocity normal impact (Figure 9) with a $2 \mathrm{~mm}$ diameter alumina particle and for an oblique impact at $60^{\circ}$ with the same type of particle with an initial velocity equal to $60 \mathrm{~m} / \mathrm{s}$ (Figure 10). As observed from experiments, see Figures 11 and 12, the present numerical model predicts that delamination does not occur under the particle. This is due to this region being subjected to high normal compressive stresses. The debonding criterion given by Equation [24] is therefore not reached.

Figure 9 shows the evolution of radial stresses in the structure at different normal impact times. At the beginning of the impact, the paint delamination is initiated. When radial compressive stresses are enough to induce paint buckling, the paint is buckled and the delamination then propagates till the particle rebounds.

Figure 10 shows the evolution of radial stresses in the paint and substrate at different times for oblique impacts. The paint delamination is initiated around the limit of the contact region between the particle and paint, but the delamination propagates only from the side in front of the particle. In fact in this region the radial stresses in the paint are in compression and higher than in the region behind the particle. The paint buckling then occurs only in a region in front of the particle.

We have compared the dimensions of the delaminated region issued from measurements using micrographies (from an optical microscope) and numerical simulations. We found that in normal impact the diameter of the delaminated region after experiments (repeated 5 times) was $2 \mathrm{~mm}$ and based on simulations was $2.3 \mathrm{~mm}$, which is near the experimental value. However in oblique impacts we noticed that the delaminated region is overestimated relative to our model. 

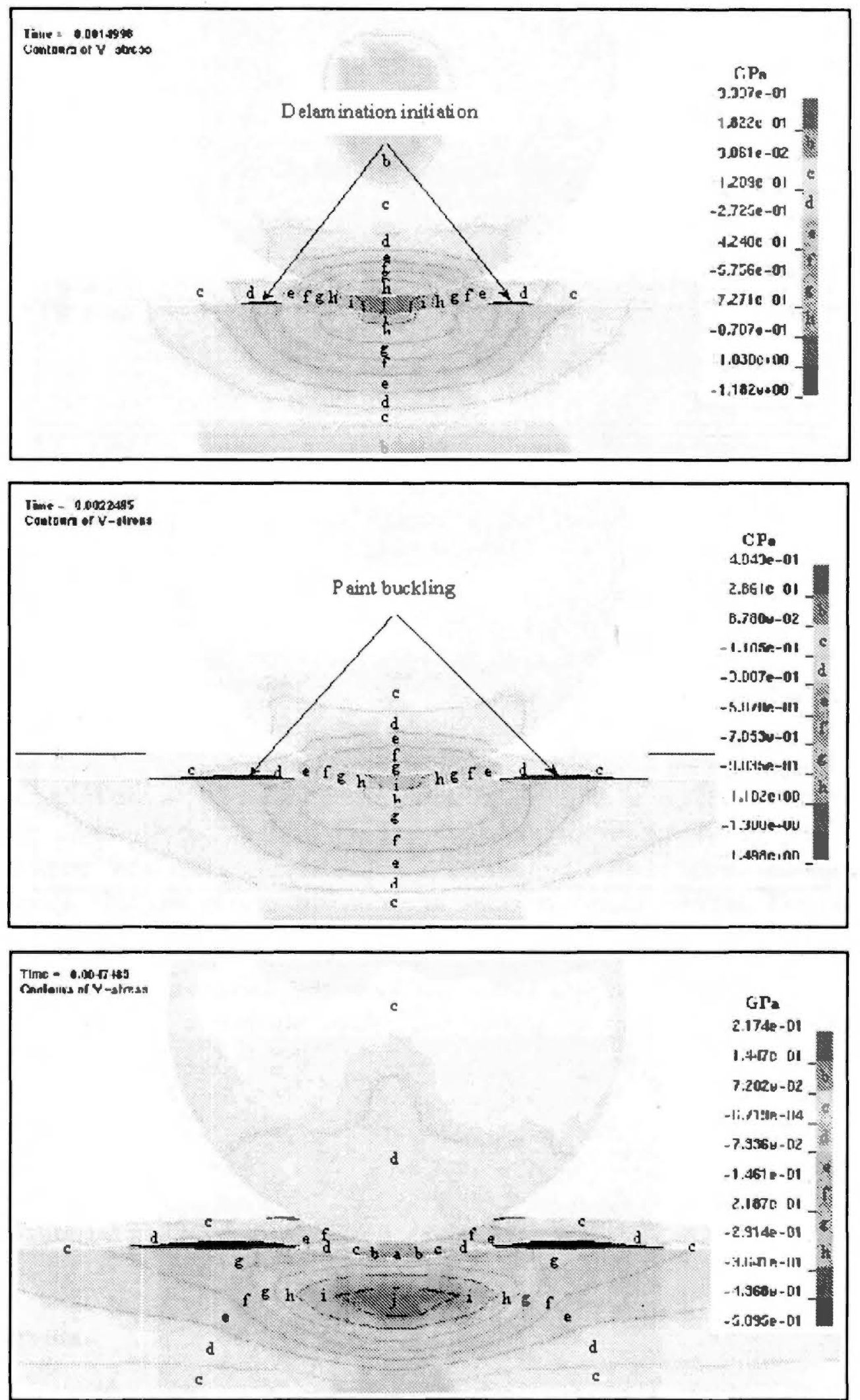

Figure 9. Paint delamination evolution in the case of normal impact $(V=81 \mathrm{~m} / \mathrm{s})$ 


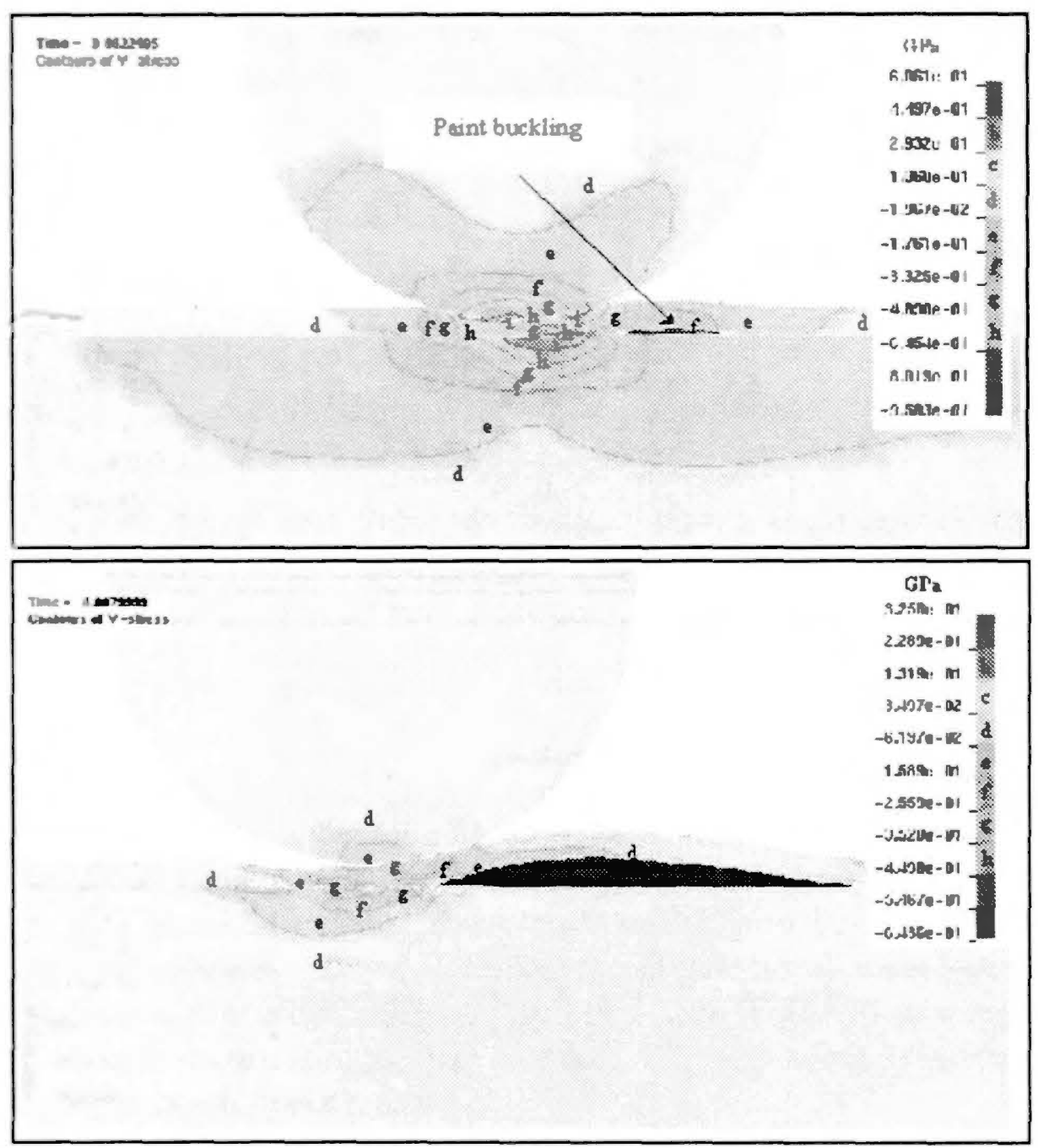

Figure 10. Paint delamination evolution in the case of oblique impact $(V=60 \mathrm{~m} / \mathrm{s})$

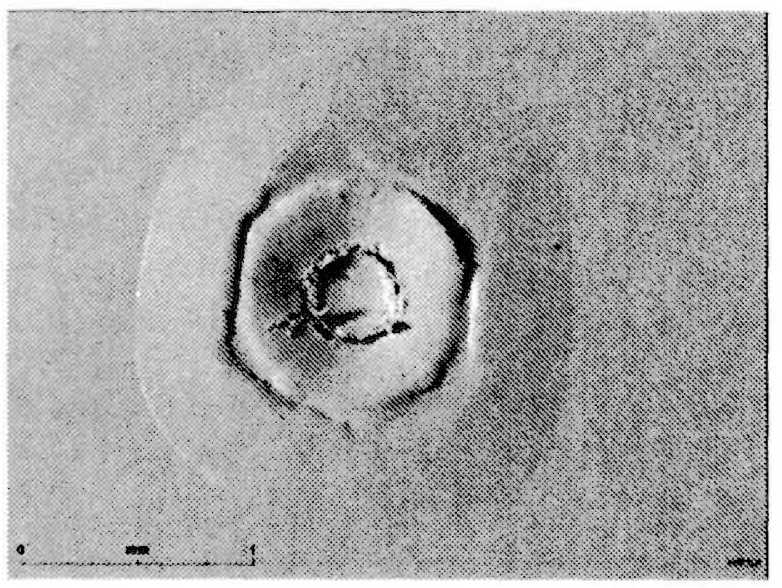

Figure 11. Normal impact with a $2 \mathrm{~mm}$ diameter alumina particle $(V=81 \mathrm{~m} / \mathrm{s})$ 


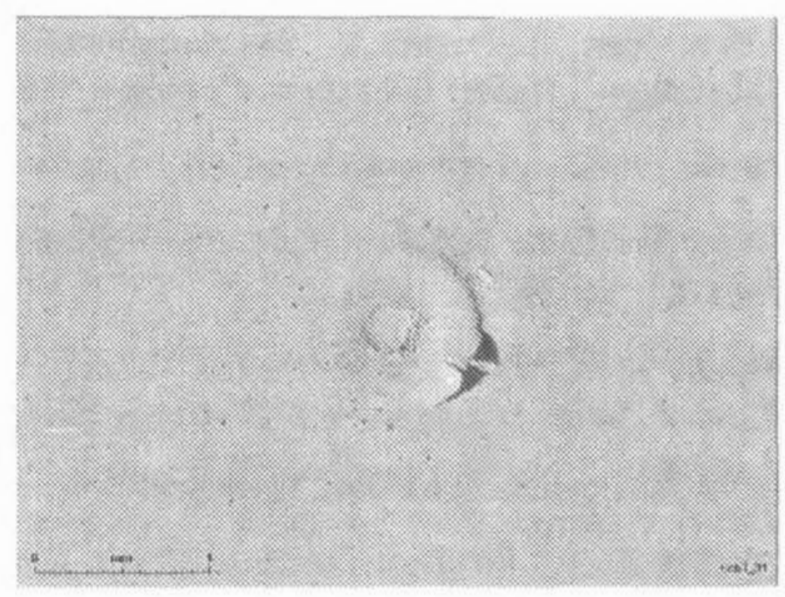

Figure 12. Oblique impact at $60^{\circ}$ with a $2 \mathrm{~mm}$ diameter alumina particle $(V=60 \mathrm{~m} / \mathrm{s})$

\section{Conclusion}

The identification of material parameters of a behaviour law by solving an inverse problem is a very efficient technique, especially when there is no experimental procedure to give direct values of these parameters. We have used this technique to identify the coefficients of the behaviour law of a paint layer in the case of impacts. We notice that the difficulties are in finding the best minimisation technique of the objective function. The use of the Levenberg-Marquardt method presents some weaknesses, for instance the need of derivatives which are computed numerically. This can induce difficulties in the convergence process. The use of a non deterministic method as genetic algorithm can bring some improvements.

The use of a constitutive behaviour law taking into account the strain rates is essential to having a realistic model and results of the impact problem. The establishment of numerical data for the paint constitutive law has enabled us to achieve a numerical simulation of the paint delamination. These simulations confirm that the paint delamination is initiated by shear stresses at the beginning of the impact. When the particle penetrates through the coating, the radial stresses are compressive and induce paint buckling when they reach a critical value. Paint delamination propagates following a mixed mode.

\section{References}

Bathe K. J., Finite element procedures, Prentice-Hall 1996.

Bonnet M., «Le problème mathématique de l'inversion : généralités, solutions exactes », in Problèmes inverses de l'expérimentation à la modélisation, OFTA, Paris, octobre 1999, p. 41-58. 
Brown M., "Yield Behaviour of Polymers", in Failure of Plastics, Brostow, W., Corneliussen, R. D., (Editors), Hanser Publishers, Chapter 6, 1986, p. 98-118.

Bui H. D., Introduction aux problèmes inverses en mécanique des matériaux, Eyrolles, 1993.

Carroll C. W., "The created response surface technique for optimizing non-linear restrained systems", Operations Res., 9, 1961, p. 169-184.

Evans A. G., Hutchinson J. W., "On the mechanics of delamination and spalling in compressed films", Int. J. Solids Structures, vol. 20, No. 5, 1984, p. 455-466.

Hallquist J. O., LS-DYNA3D Theoretical Manual, Livermore Software Technology, 1998.

Hughes T. J. R., Belytschko T., "A Precis of Developments in Computational Methods for Transient Analysis", Journal of Applied Mechanics, vol. 50, 1983, p. 1033-1041.

Johnson G. R., Cook W. H., "A Constitutive Model and Data for Metals Subjected to Large Strains, High Strain Rates and High Temperatures", $7^{\text {th }}$ Int Symposium on Ballistics, The Hague, 1983, p. 541-547.

Kolsky H., Stress Waves in Solids, Dover, New York, 1963.

Komvopoulos K., "Finite Element Analysis of a Layered Elastic Solid in Normal Contact with a Rigid Surface", ASME journal of tribology, vol. 110, 1998, p. 477-485.

Kral F. R., Komvopoulos K., Bogy D. B., "Finite Element Analysis of Repeated Indentation of an Elastic-Plastic Layered Medium by a Rigid Sphere, part I: Surface Results", $A S M E$ journal of applied mechanics, vol. 62, 1995a, p. 20-28.

Kral F. R., Komvopoulos K., Bogy D. B., "Finite Element Analysis of Repeated Indentation of an Elastic-Plastic Layered Medium by a Rigid Sphere, part II: Subsurface Results", ASME journal of applied mechanics, vol. 62, 1995b, p. 29-42.

Marquardt D. W., "An algorithm for least-squares estimation of nonlinear parameters", J. Soc. Indust. Appl. Math., vol. 11, No. 2, June, 1963, p. 431-441.

Marusich T. D., Ortiz M., "Modelling of High-Speed Machining”, International Journal for Numerical Methods in Engineering, vol. 38, 1995, p. 3675-3694.

Massoni E., "Identification automatique du comportement par analyse inverse », Séminaire de plasticité et mise en forme des métaux, Chapitre 20, CEMEF, Ecole des Mines de Paris 1994.

Monnette D. J. L., Oestreich J., "New developments in starch media applications and coating removal effects on various aerospace materials", in Proceedings of the 1996 DOD/Industry Advanced Coating Removal Conference, Batelle Memorial Institute, Lake Buena Vista, FL, 1996.

Papini M., Spelt J. K., "Organic Coating Removal by Particle Impact", Wear 213, 1997, p. 185-199.

Papini M., Spelt J. K., "The Plowing Erosion of Organic Coatings by Spherical Particles", Wear 222, 1998, p. 38-48.

Schnur D. S., Zabaras N., "An inverse method for determining elastic material properties and a material interface", International Journal for Numerical methods in Engineering, vol. 33, 1992, p. 2039-2057. 
Tarantola A., Inverse problem theory, Elsevier, 1987.

Van Krevelen D. W., "Thermophysical Properties of Polymers", in Properties of Polymers, Elsevier, 1990, p. 71-286.

Yin W. L., "Axisymmetric buckling and growth of a circular delamination in a compressed laminate”, Int. J. Solids Structures, 1985, vol. 21, No. 5, p. 503-514.

Zhao H., "Testing of Polymeric Foams at High and Medium Strain Rates", Polymer Testing, vol. 16, 1997, p. 507-516.

Zouari B., Touratier M., "Numerical Simulation of Aeronautical Paint Layer Decoating", The $5^{\text {th }}$ International Conference on Computational Structures Technology, 6-8 September 2000, Leuven, Belgium, vol. Finite Elements: Techniques and Developments, p. 231-236.

Zouari B., Modélisation et Simulation de l'Enlèvement de Revêtements Polymériques par Impact Particulaire, Thèse de doctorat, Ecole Nationale Supérieure d'Arts et Métiers-CER Paris, Juin 2001.

Zouari B., Touratier M., "Simulation of organic coating removal by particle impact", Wear 253/3-4, 2002, p. 488-497. 